\title{
MODELLING OF THERMAL DAMAGE IN LASER IRRADIATED TISSUE
}

\author{
Marek Jasiński \\ Institute of Computational Mechanics and Engineering \\ Silesian University of Technology \\ Gliwice, Poland \\ marek.jasinski@polsl.pl
}

\begin{abstract}
In the paper the numerical analysis of thermal processes proceeding in the 2D homogeneous biological tissue subjected to laser irradiation is presented. In particular, the influence of necrotic changes in tissue on the values of the perfusion coefficient and effective scattering coefficient are discussed. The transient heat transfer is described by the bioheat transfer equation in the Pennes formulation. As a model of light distribution in tissue, the first-order scattering approach has been used. At the stage of numerical realization the $1^{\text {st }}$ scheme of the boundary element method has been applied.
\end{abstract}

Keywords: bioheat transfer, Arrhenius scheme, tissue damage, laser irradiation

\section{Introduction}

From the thermal point of view, the interaction between the laser beam and the biological tissue causes a local increase in the temperature field value, which can lead to a number of changes in the structure of the tissue. Depending on the degree of the temperature's change a decrease may occur in the activity of enzymes, denaturation of proteins and collagen or the increase in membrane permeability and vaporization. The extreme case of interaction is tissue charring. On the other hand, in cases where the temperature rise is moderate, the only tissue reaction is vasodilatation, which is not accompanied by necrosis $[1,2]$.

It is obvious that the destruction of the tissue is reflected in the values of its parameters. The perfusion coefficient is very often treated as the main indicator of tissue injury, thus in the literature there are some relations between temperature and perfusion as well as the degree of tissue damage and perfusion [3].

In the case of photothermal reaction, it is also possible to link the tissue damage with changes in the values of optical parameters of tissue. It is known that practically all soft tissues have strong scattering characteristics and, due to denaturation, the phenomenon of "whitening" as a result in scattering increases in the tissue can be observed [1]. Based on the knowledge of scattering coefficient both native 
as well as denaturated tissue in [4] the exponential function in order to investigate changes in tissue scattering during laser-tissue interaction has been used.

\section{Governing equations}

The problem that has been taken into consideration was an interaction between laser beam and the 2D domain of homogeneous biological tissue of a rectangular shape, as is shown in Figure 1. The transient heat transfer is described by the Pennes formulation in the form [3-11]

$$
\mathbf{x} \in \Omega: \quad c \dot{T}=\lambda T_{, i i}+Q_{V}
$$

where $\lambda\left[\mathrm{Wm}^{-1} \mathrm{~K}^{-1}\right]$ is the thermal conductivity, $c\left[\mathrm{Jm}^{-3} \mathrm{~K}^{-1}\right]$ is the volumetric specific heat, $T=T(\mathbf{x}, t)$ is the temperature while $\dot{T}$ denotes its time derivative. The component $Q_{V}\left[\mathrm{Wm}^{-3}\right]$ is the internal heat source containing information connected with the perfusion, metabolism and laser irradiation:

$$
Q_{V}(\mathbf{x}, t)=Q_{\text {perf }}(\mathbf{x}, t)+Q_{\text {las }}(\mathbf{x}, t)+Q_{m e t}(\mathbf{x}, t)
$$

The metabolic heat source $Q_{m e t}\left[\mathrm{Wm}^{-3}\right]$ is assumed as a constant value, while the perfusion heat source is defined as

$$
Q_{\text {perf }}(\mathbf{x}, t)=c_{B} G_{B}\left[T_{B}-T(\mathbf{x}, t)\right]
$$

where $G_{B}\left[\left(\mathrm{~m}^{3}{ }_{\text {blood }} / \mathrm{s}\right) /\left(\mathrm{m}^{3}\right.\right.$ tissue $\left.)\right]$ is the perfusion coefficient, $c_{B}\left[\mathrm{Jm}^{-3} \mathrm{~K}^{-1}\right]$ is the volumetric specific heat of blood and $T_{B}$ corresponds to the artery temperature $[3,7,12]$.

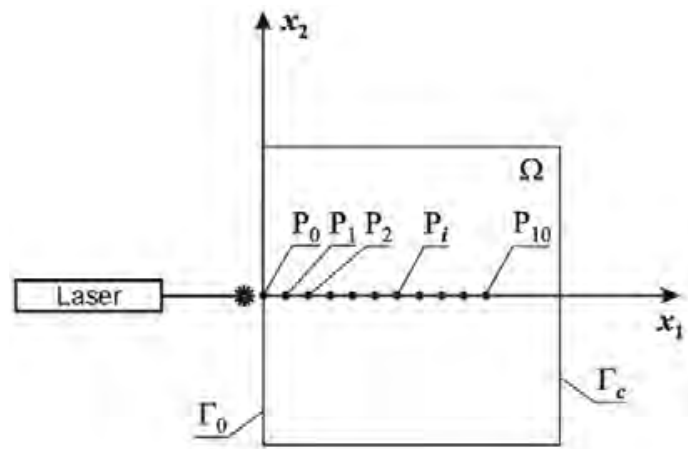

Fig. 1. The domain considered

It is well known that biological tissues are characterized by a strong scattering and weak absorption in the so-called therapeutic window. Moreover, during the process of coagulation, the changes in tissues lead to higher scattering, while the absorption 
remains the same. In such kinds of cases the radiative transfer calculation is usually used to modelling of tissue-laser interactions, however, sometimes in simulations of laser thermotherapy only tissue absorption is considered [1, 4].

The first-order scattering method is the simplest approach which allows one to take into account the scattering properties of tissue. Its definition is based on the Beer-Lambert law, meaning that radiation is treated in a similar way as absorbed light. It should be pointed out that in many applications, the use of this approach may lead to errors in estimation of the absorbed radiation, but on the other hand, it could be useful in some cases, similar to those analyzed in the current paper.

So, the laser heat source is assumed as $[1,4,11]$

$$
Q_{\text {las }}(\mathbf{x}, t)=\mu_{t}^{\prime} I_{0} \exp \left(-\frac{2 r^{2}}{w^{2}}\right) \exp \left(-\mu_{t}^{\prime} \mathbf{x}\right) p(t)
$$

where $I_{0}\left[\mathrm{~W} \mathrm{~cm}^{-2}\right]$ is the surface irradiance of laser, $w$ is the radius of laser beam, $r$ is the distance between the point and the main axis of the beam and $p(t)$ is the function equal to 1 when the laser is on and equal to 0 when the laser is off, while $\mu_{t}^{\prime}\left[\mathrm{cm}^{-1}\right]$ is the attenuation coefficient defined as

$$
\mu_{t}^{\prime}=\mu_{a}+\mu_{s}^{\prime}
$$

where $\mu_{a}\left[\mathrm{~cm}^{-1}\right]$ is the absorption coefficient and $\mu_{s}^{\prime}\left[\mathrm{cm}^{-1}\right]$ denotes the effective scattering coefficient $[1,4,11]$.

The bioheat transfer equation (1) is supplemented by the Robin condition assumed on the external boundary of tissue subjected to laser irradiation $\Gamma_{0}$

$$
\mathbf{x} \in \Gamma_{0}: \quad q(\mathbf{x}, t)=\alpha\left(T-T_{a m b}\right)
$$

where $\alpha\left[\mathrm{Wm}^{-2} \mathrm{~K}^{-1}\right]$ is the convective heat transfer coefficient and $T_{a m b}$ is the temperature of surroundings while on the remaining parts of boundary $\left(\Gamma_{c}\right)$ the no-flux condition is accepted and the initial distribution of temperature is also known.

\section{Thermal damage of the tissue}

Thermal damage of the tissue could be described by the Arrhenius injury integral $[2-5,9]$

$$
\theta(\mathbf{x})=\int_{0}^{t^{F}} A \exp \left[-\frac{\Delta E}{R T}\right] \mathrm{d} t
$$

where $R$ is the universal gas constant $\left[\mathrm{J} \mathrm{mole}^{-1} \mathrm{~K}^{-1}\right], \Delta E$ is the activation energy $\left[\mathrm{J}\right.$ mole $\left.{ }^{-1}\right]$ and $A$ is the pre-exponential factor $\left[\mathrm{s}^{-1}\right],\left[0, t^{F}\right]$ is the considered time interval. It should be pointed out that the criterion of tissue damage is assumed as 


$$
\theta(\mathbf{x}, t) \geq 1
$$

The main assumption of the Arrhenius scheme is that the tissue thermal damage is irreversible, but it is also known that, when the rise in temperature is moderate, the blood vessels in tissue become dilated without being thermally damaged.

In order to model the possibility of withdrawal of tissue injury, when the thermal interaction of external heat source or laser irradiation is ceased, the following algorithm is introduced (Fig. 2) [7].

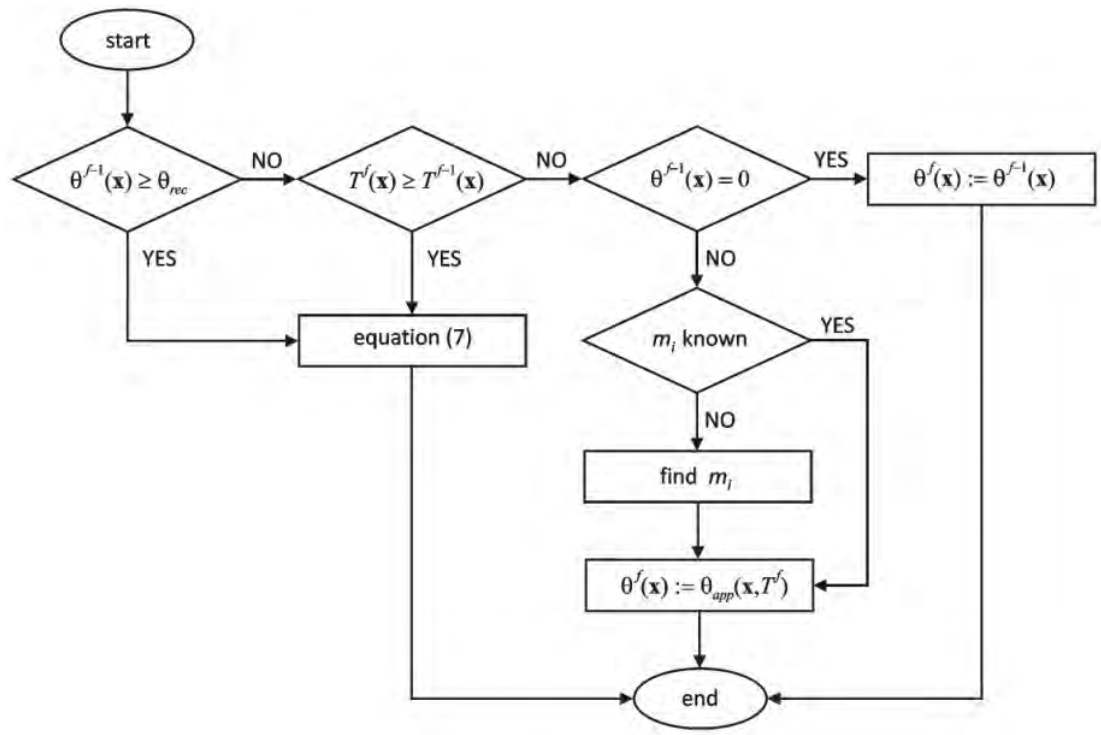

Fig. 2. The algorithm of tissue injury calculation

Firstly, the recovery threshold denotes as $\theta_{\text {rec }}$ is accepted. The withdrawal of tissue destruction will be possible only for those points of the domain considered in which the value of the injury integral is below the recovery threshold. If the injury integral at the point $\mathbf{x} \in \Omega$ achieves the value equal to or greater than $\theta_{\text {rec }}$ then injury of the tissue becomes irreversible (so, it will be calculated using equation (7)).

Then, let us assume that for the time interval $\left[0, t^{F}\right]$ being under consideration and divided into $F$ subintervals $\left[t^{f-1}, t^{f}\right]$ (where $f=1,2 \ldots F$ ), both the values $T^{0}(\mathbf{x})$ $\ldots T^{f}(\mathbf{x})$ and $\theta^{0}(\mathbf{x}) \ldots \theta^{f-1}(\mathbf{x})$ at the point $\mathbf{x} \in \Omega$ are known.

In the case when for time $t^{f}$ the condition $\theta^{f}(\mathbf{x})<\theta_{\text {rec }}$ is satisfied, the function denoted as $\theta_{a p p}(\mathbf{x}, T)$ is introduced in order to model of the withdrawal of the tissue injury. In the current paper it is assumed as the linear one between $\left(T^{k-1}, \theta^{k-1}\right)$ and $\left(T^{f-1}, \theta^{f-1}\right)$.

The upper index $k$ refers to the first time step in which the elevation of temperature at the point $\mathbf{x}$ was noticed. For the time $t^{f+1}$, if $T^{f+1}<T^{f}$ and $\theta^{f}<\theta_{\text {rec }}$ again the function $\theta_{\text {app }}$ defined for time $t^{f}$ is used. 
In order to model the necrotic changes in the domain of tissue considered some parameters are assumed to be tissue damage-dependent.

The equation describing the dependence between tissue damage and perfusion should reproduce the initial increase in perfusion (vasodilatation) and its subsequent decline corresponding to the vasculature closing as well. So, the perfusion coefficient can be assumed in the form of polynomial function of the $2^{\text {nd }}$ order [3, 7]

$$
G_{B}(\theta)=G_{B 0} \sum_{j=1}^{3} b_{j} \theta^{j-1}
$$

where $G_{B 0}$ is the initial perfusion coefficient and $b_{j}$ are the function coefficients.

As it was said previously, the scattering in tissue is increasing with the increasing of the tissue coagulation. Thus, the effective scattering coefficient is described as

$$
\mu_{s}^{\prime}(\theta)=\mu_{\text {snat }}^{\prime} \exp (-\theta)+\mu_{\text {sden }}^{\prime}[1-\exp (-\theta)]
$$

where $\mu_{\text {snat }}^{\prime}$ and $\mu_{s d e n}^{\prime}\left[\mathrm{cm}^{-1}\right]$ denote the effective scattering coefficient for native and destructed (denaturated) tissue, respectively [4].

\section{Results}

The problem described by equation (1) has been solved using the $1^{\text {st }}$ scheme of the BEM for 2D transient heat diffusion $[10,11]$.

As mentioned earlier, the aim of the research is to analyze the destructive changes in 2D tissue domain of size $4 \times 4 \mathrm{~cm}$ during laser irradiation (Fig. 1). The interior of domain has been divided into 1600 internal constant cells, while the external boundary has been divided into 160 constant elements.

The thermophysical and optical properties, as well as the parameters of Arrhenius injury integral used in simulations, are collected in Table 1. It should be pointed out that optical parameters $\mu_{a}, \mu_{\text {snat }}^{\prime}$ and $\mu_{s d e n}^{\prime}$ are typical for near - IR irradiation on soft tissue (e.g. Nd:YAG laser of $1064 \mathrm{~nm}$ used for prostate coagulation). In this kind of laser - tissue interaction during coagulation the reduced scattering coefficient increases up to $300 \div 400 \%$ of initial coefficient value (i.e. effective scattering coefficient of native tissue) while the absorption coefficient remains on the same level [4].

In Table 2, the values of coefficients of polynomial function describing relation between tissue destruction and perfusion coefficient (cf. equation (9)) are shown. The values of coefficients for the interval from 0 to 0.1 respond to the increase of the perfusion coefficient caused by vasodilatation up to the value $\theta=0.05$ (maximum of the function and the recovery threshold $\theta_{\text {rec }}$ ) and the beginning of the narrowing of blood vessels, while for the interval 0.1 to 1 they reflect blood flow decrease as the vasculature is going to shut down (thrombosis) [3]. 
Thermophysical and optical properties of tissue and blood, Arrhenius injury integral parameters $[4,7,11]$

\begin{tabular}{|c|c|c|c|}
\hline$\lambda$ & Thermal conductivity of tissue & 0.609 & $\mathrm{Wm}^{-1} \mathrm{~K}^{-1}$ \\
\hline$c$ & Volumetric specific heat of tissue & 4.18 & $\mathrm{MJm}^{-3} \mathrm{~K}^{-1}$ \\
\hline$G_{B 0}$ & Initial blood perfusion coefficient & 0.00125 & $\mathrm{~s}^{-1}$ \\
\hline$\mu_{a}$ & Absorption coefficient of tissue & 0.4 & $\mathrm{~cm}^{-1}$ \\
\hline$\mu_{\text {snat }}^{\prime}$ & Effective scattering coefficient of native tissue & 10 & $\mathrm{~cm}^{-1}$ \\
\hline$\mu_{\text {sden }}^{\prime}$ & Effective scattering coefficient of destructed tissue & 40 & $\mathrm{~cm}^{-1}$ \\
\hline$Q_{m e t}$ & Metabolic heat source & 245 & $\mathrm{Wm}^{-3}$ \\
\hline$c_{B}$ & Volumetric specific heat of blood & 3.9962 & $\mathrm{MJm}^{-3} \mathrm{~K}^{-1}$ \\
\hline$T_{B}$ & Arterial blood temperature & 37 & ${ }^{\circ} \mathrm{C}$ \\
\hline$A$ & Pre-exponential factor & $3.1 \times 10^{98}$ & $\mathrm{~s}^{-1}$ \\
\hline$\Delta E$ & Activation energy & $6.27 \times 10^{5}$ & $\mathrm{~J} \mathrm{~mole}^{-1}$ \\
\hline$R$ & Universal gas constant & 0.314 & $\mathrm{~J} \mathrm{~mole}^{-1} \mathrm{~K}^{-1}$ \\
\hline$\theta_{\text {rec }}$ & Recovery threshold & \\
\hline
\end{tabular}

The coefficients of $G_{B}(\theta)$ function (cf. equation (9)) [3]

Table 2

\begin{tabular}{|c|c|c|c|}
\hline & $b_{1}$ & $b_{2}$ & $b_{3}$ \\
\hline$\theta=0$ & 1 & 0 & 0 \\
\hline $0<\theta \leq 0.1$ & 1 & 25 & -260 \\
\hline $0.1<\theta \leq 1$ & 1 & -1 & 0 \\
\hline$\theta>1$ & 0 & 0 & 0 \\
\hline
\end{tabular}

In Table 3 the parameters of boundary-initial conditions and the laser parameters are presented. It should be noted that there is a wide range of laser parameters, which are applicable for near-IR irradiation of soft tissue. Due to this, the set of parameters presented in table is only one of possible options. Moreover, the different types of laser impulses are used. Because of that, the goal of the current paper is to compare how the different types of impulses affect to the process of thermal damage of tissue.

The two cases of laser irradiation were taken under consideration: the multiple laser impulse ( 100 seconds on and 100 seconds off $)$ in the $1^{\text {st }}$ example while in the $2^{\text {nd }}$ example the laser is controlled by surface temperature, that means the laser is on when the temperature drops below $T_{\text {ctrl }}-\Delta T_{\text {ctrl }}$ and off when the temperature reaches above $T_{c t r l}+\Delta T_{c t r l}$.

The results containing the information about courses in time are presented in selected points $\mathrm{P}_{0}, \mathrm{P}_{1} \ldots . \mathrm{P}_{10}$ which are placed on the main optical path of the laser beam (cf. Fig. 1), so their co-ordinates are $\mathrm{P}_{0}(0,0), \mathrm{P}_{1}(0.0005,0), \mathrm{P}_{2}(0.0015,0)$, ..., $\mathrm{P}_{10}(0.0095,0)$. 
Table 3

Parameters of boundary-initial conditions and laser $[4,11,14]$

\begin{tabular}{|c|c|c|c|}
\hline$I_{0}$ & Surface irradiance of laser & 3 & $\mathrm{~W} \mathrm{~cm}^{-2}$ \\
\hline$d$ & Laser beam diameter & 2 & $\mathrm{Mm}$ \\
\hline$\alpha$ & Convective heat transfer coefficient & 10 & $\mathrm{~W} \mathrm{~m}^{-2} \mathrm{~K}^{-1}$ \\
\hline$T_{a m b}$ & Ambient temperature & 20 & ${ }^{\circ} \mathrm{C}$ \\
\hline$T_{p}$ & Initial temperature of tissue & 37 & ${ }^{\circ} \mathrm{C}$ \\
\hline$T_{c t r l} \pm \Delta T_{c t r l}$ & Controlled temperature in example 2 & $70 \pm 3$ & ${ }^{\circ} \mathrm{C}$ \\
\hline
\end{tabular}

Figures 3 and 4 are associated with the multiple laser impulse case. In Figure 3 the time courses of temperature and the injury integral $\theta$ are shown. It is visible that for each of the subsequent laser's cycle (on/off) the reached temperatures are higher and higher. For the Arrhenius integral the influence of the proposed algorithm of injury calculation at the points $\mathrm{P}_{5}$ and $\mathrm{P}_{7}$ could be noted. In the next figure the courses of tissue damage-dependent parameters are shown. The impact of the value of injury integral on the values of these parameters is visible both for the perfusion coefficient and the effective scattering coefficient.
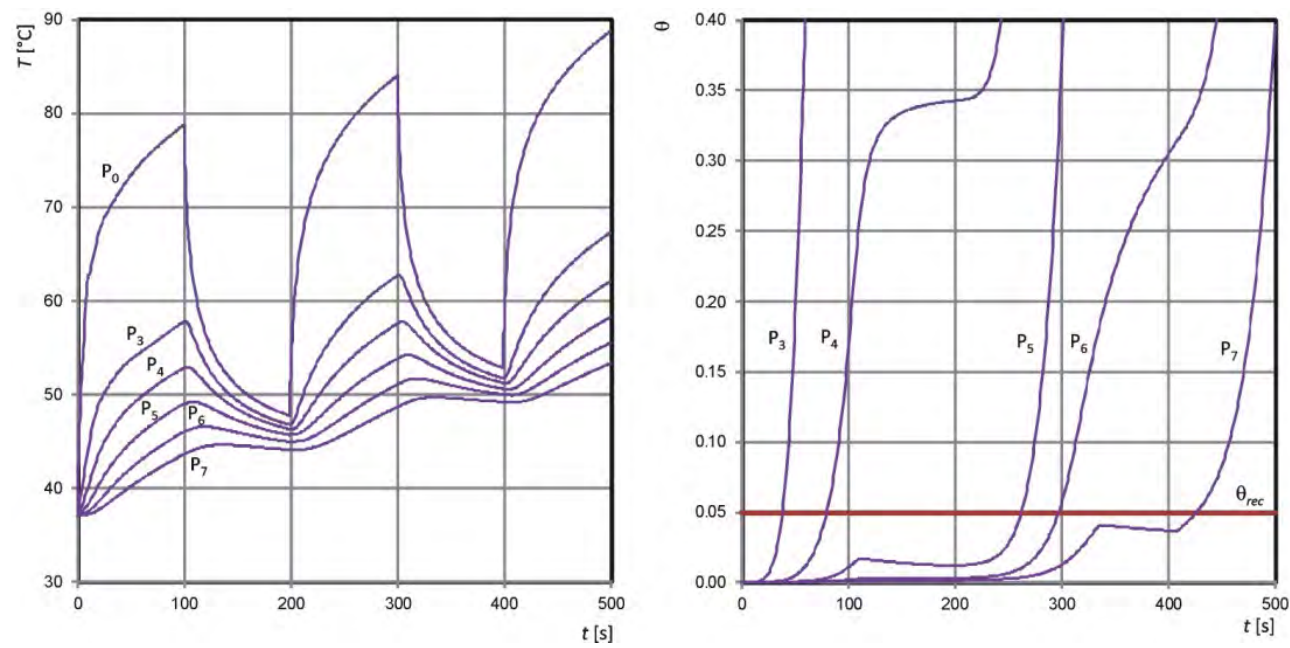

Fig. 3. Courses of temperature and the injury integral $\theta$ (example 1)

The results obtained in the $2^{\text {nd }}$ example of computations are presented in Figures 5 and 6. Figure 5 concerns the time courses of temperatures and the Arrhenius integral, while Figure 6 shows information about time courses of the perfusion coefficient and effective scattering coefficient. The decrease of the Arrhenius integral value was noticed several times (point $\mathrm{P}_{4}$ ), according to the injury calculation algorithm. Although the declines are not big, they are clearly visible, just as its 
influence on the values of the perfusion coefficient and the effective scattering coefficient.
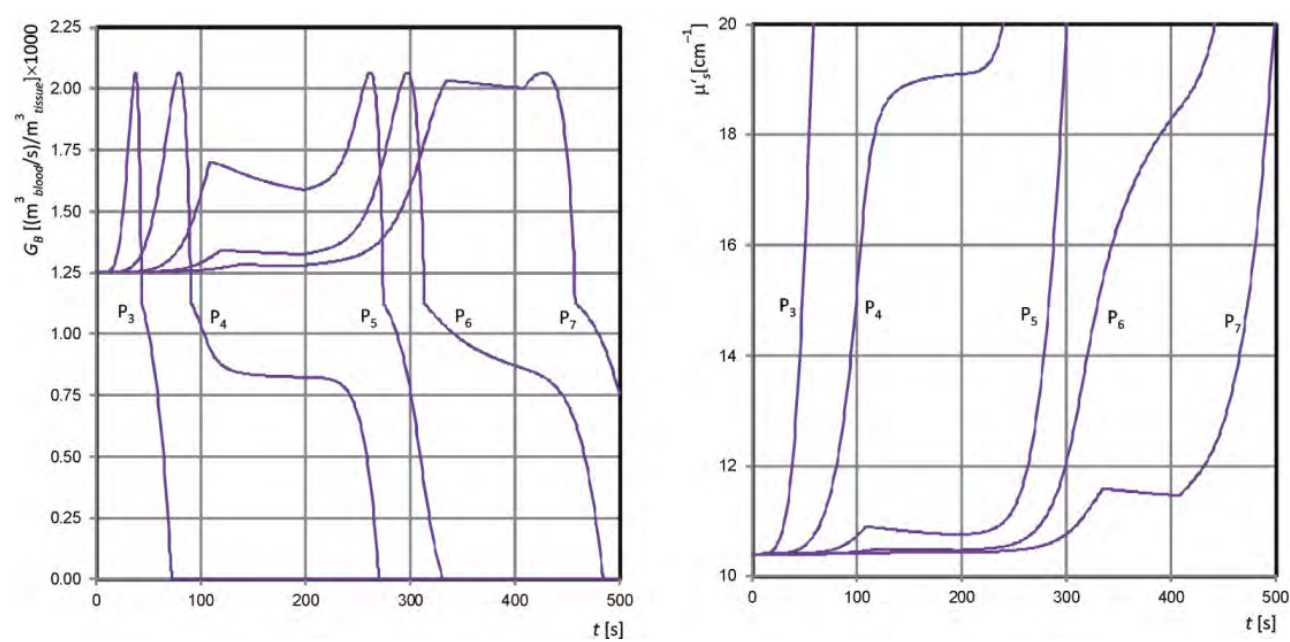

Fig. 4. Courses of the perfusion coefficient $G_{B}$ and the effective scattering coefficient $\mu_{s}^{\prime}$ (example 1)
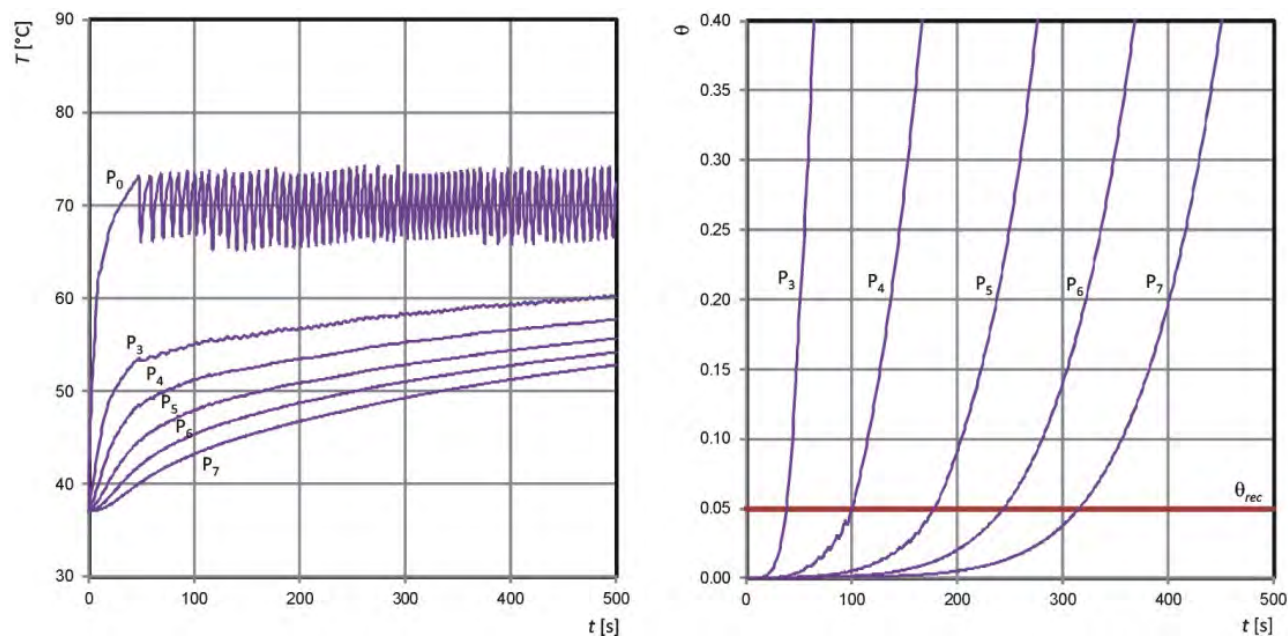

Fig. 5. Courses of temperature and the injury integral $\theta$ (example 2)

In Figure 7 the information on proliferation of the lesion is presented. The number of elements on the scale of the figure means that tissue injury is treated as a sum of the elements on which the injury integral is above 0.01 , so this value could be treated as the border of thermally untouched tissue. 

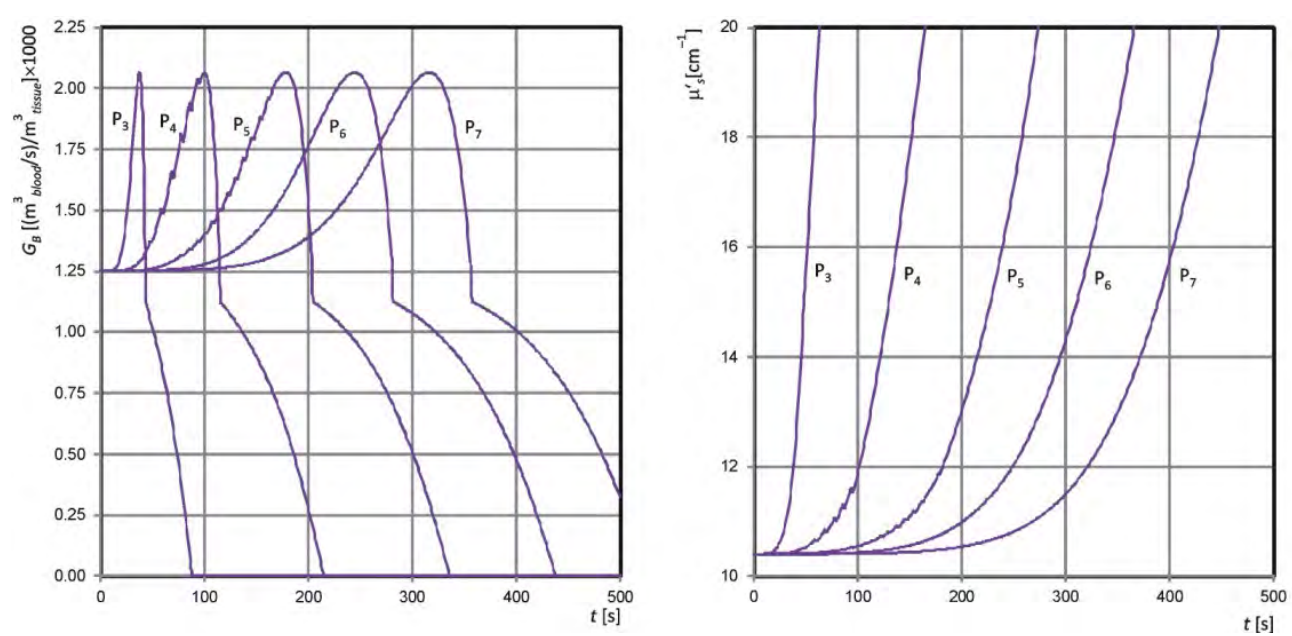

Fig. 6. Courses of the perfusion coefficient $G_{B}$ and the effective scattering coefficient $\mu_{s}^{\prime}$ (example 2)

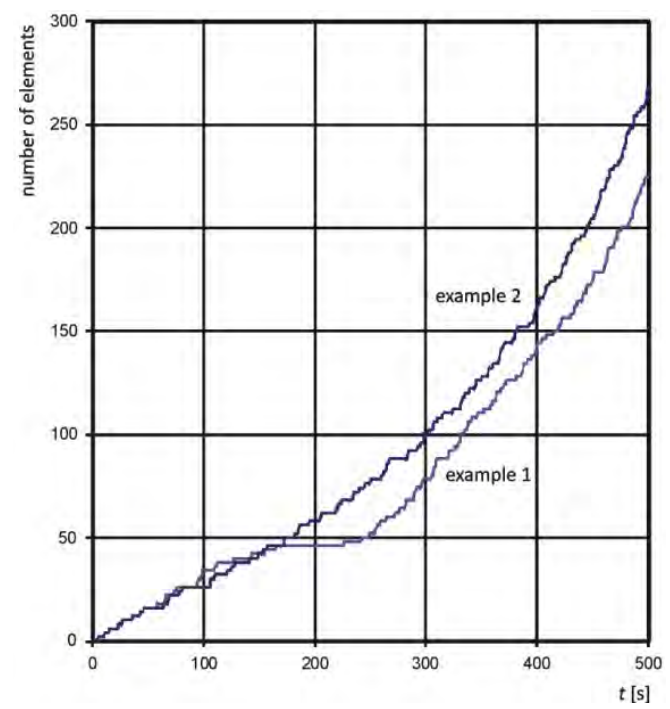

Fig. 7. Proliferation of thermal injury

In Figure 8 the distributions of injury integral after 100, 300 and 500 seconds are presented. In fact, this figure should be treated as an illustration of the shape of the thermal damage of tissue. The white zone in the figures refers to the values of injury integral below the recovery threshold $\left(\theta_{\text {rec }}=0.05\right)$, the grey zone refers to the values $0.05<\theta<1$ so it's a partial damage area, and the black zone illustrates the area in which Arrhenius integral achieved the criterion of tissue necrosis (cf. equation (8)). 

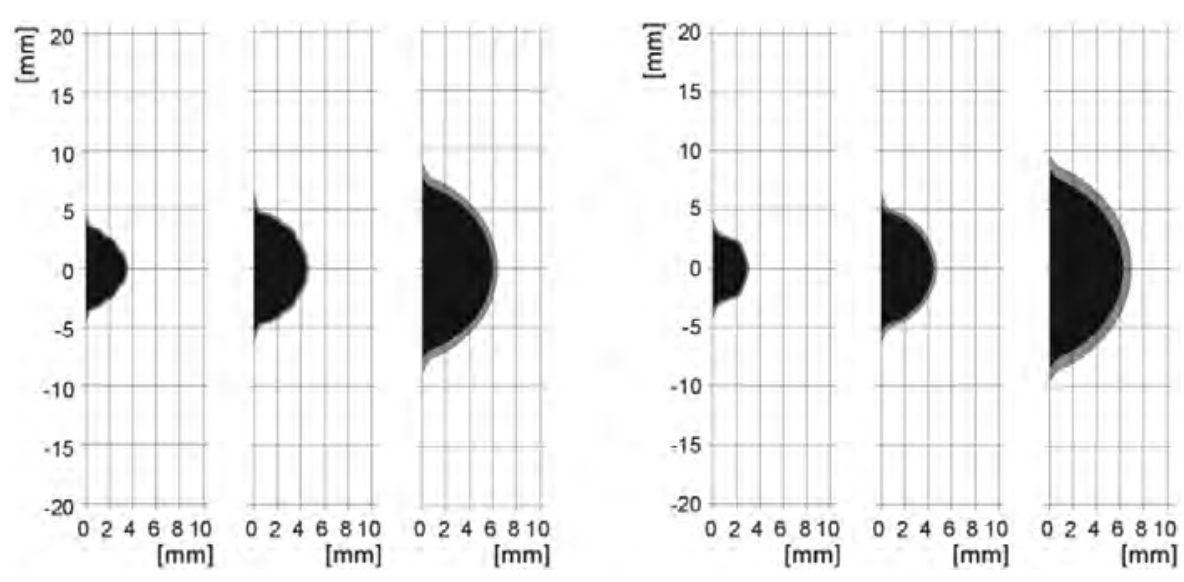

Fig. 8. Distribution of injury integral after 100, 300 and 500 seconds in example 1 (LHS) and example 2 (RHS)

\section{Discussion}

In the two analyzed cases of tissue subjected to laser irradiation, the points in which the process of withdrawal of tissue injury according to the algorithm proposed are noticeable. It is worth noting that in some of them, e.g. at the points $\mathrm{P}_{5}$ and $\mathrm{P}_{7}$ in the example of multiple laser impulse (Fig. 3), the decrease of the Arrhenius integral value was temporary, then in the next cycle, when the laser is on, the value of integral is above the recovery threshold. Finally, the injury reached the necrosis criterion (at the point $\mathrm{P}_{5}$ after $332 \mathrm{~s}$ ) or stabilized at a level corresponding to the partially damaged tissue (at the point $\mathrm{P}_{7}$ after $500 \mathrm{~s}: \theta=0.4$ ).

In example 2, that means the case in which the laser is controlled by the temperature at the surface of tissue, the withdrawal of the injury is also visible (mainly courses at the points $\mathrm{P}_{4}$ and $\mathrm{P}_{5}$ - Fig. 5) but, due to the nature of laser irradiation, in this example the effect of withdrawal is short-term and does not significantly decrease the value of the injury integral.

Obviously, all changes in the value of the Arrhenius integral have reflection in changes of tissue damage - dependent parameters assumed in the current model, i.e. perfusion coefficient and effective scattering coefficient. While for the effective scattering coefficient, in principle, an increase of the value of integral leads to an increase in the value of the scattering coefficient, for the perfusion coefficient increase is only up to the recovery threshold. The coagulation zone is often adjacent to the area of raised value of perfusion (so-called hyperemic ring) [1, 4], as can be seen by comparing the curves for selected time steps. For example, in the multiple laser impulse case (Fig. 4), for the time $200 \mathrm{~s}$ the perfusion coefficient value at point $\mathrm{P}_{4}$ is about $0.008 \mathrm{~s}^{-1}$, which is reduced value in relation to the initial perfusion coefficient $G_{B 0}$ (partial - coagulation area), while at the point $\mathrm{P}_{5}$ lying in its vicinity this value is equal to $G_{B}=0.0015 \mathrm{~s}^{-1}$ (above $G_{B 0}$ ). 
In example 2 , the time in which the temperature decreases with a temperature from above $73^{\circ} \mathrm{C}$ to below $68^{\circ} \mathrm{C}$ is extended (the beginning of the simulation $2 \mathrm{~s}$, the end of the simulation 4 seconds) whereas time to reach $73^{\circ} \mathrm{C}$ from less than $67^{\circ} \mathrm{C}$ is reduced ( 9 seconds at the beginning of the simulation, 3 seconds at the end of the simulation). Unfortunately, this is hardly visible on the curve at point $\mathrm{P}_{0}$ in Figure 7. This is a result of the increase of the effective scattering coefficient, which shortens the path of photons in the tissue, and, in consequence, leads to greater absorption at the external surface of the tissue in the area where tissue is damaged.

Comparing the process of the increase of tissue damage, it is visible that for the second case the proliferation of the lesion occurs slightly more evenly (Fig. 7). The largest difference in the shape of the wound can be found for 100 seconds, which is closer to the beginning of the simulation. As is apparent from the course of proliferation at this stage, the area of tissue damage is greater in the case of a multiple pulse than for the case of temperature control on the surface of the tissue. Then, shapes of the thermal lesions of tissue become more and more similar to each other, although the final size of the wound is about $16 \%$ greater for example 2 (Fig. 8).

\section{Conclusions}

As the results show larger size of thermal damage was achieved at a lower local temperature value (cf. Figs. 3 and 5), which for the second example was from the scope $68 \div 73^{\circ} \mathrm{C}$, while for the case of multiple laser impulse in the third cycle (at time $500 \mathrm{~s}$ ) reached up to $90^{\circ} \mathrm{C}$.

Because the temperature is a core value to calculate injury integral this has consequences for the values that they reached. The Arrhenius integral values achieved in the $1^{\text {st }}$ example were about twice greater than for the $2^{\text {nd }}$ example. This may indicate increased tissue damage in the vicinity of the target of laser irradiation. For this reason, application of the laser impulse controlled by the surface temperature seems to be more favorable.

The proposed algorithm for calculating the thermal destruction of biological tissue based on the Arrhenius scheme allows for a more detailed estimation of the total size of the tissue destruction. In addition, one can obtain information about the area where the tissue after the termination of the laser impact can return to its native state, as well as about the size of the area of partially damaged tissue. It should be noticed that to better determine the potential size and shape of tissue damage during its interaction with the laser, the various values of thermophysical and optical parameters that may result from individual characteristics should be taken into account. For this purpose, for example, the sensitivity analysis can be used. The accuracy of the description of the light effects in the soft tissues depends on the assumed model of interaction. In the current paper aimed at analysis of the phenomenon of withdrawal of tissue thermal damage, the description of lighttissue interaction was a simple, first-order scattering model, however for a more 
realistic description the diffusion approximation or Monte Carlo model should be taken into account [1]. It is also possible to use another equation of bioheat transfer such as Cattaneo-Vernotte equation [12] or dual phase lag equation $[13,14]$.

\section{References}

[1] Niemz M.H., Laser - Tissue Interaction, Springer-Verlag, Berlin, Heidelberg, New York 2007.

[2] Henriques F.C., Studies of thermal injuries, V. The predictability and the significance of thermally induced rate process leading to irreversible epidermal injury, Arch. Pathol. 1947, 43, 489-502.

[3] Abraham J.P., Sparrow E.M., A thermal-ablation bioheat model including liquid-to-vapor phase change, pressure- and necrosis-dependent perfusion, and moisture-dependent properties, Int. J. Heat Mass Transfer 2007, 50(13-14), 2537-2544.

[4] Glenn T.N., Rastregar S., Jacques S.L., Finite element analysis of temperature controlled coagulation in laser irradiated tissue, IEEE T. Bio.-Med. Eng. 1996, 43(1), 79-86.

[5] Majchrzak E., Jasiński M., Numerical analysis of bioheat transfer processes in tissue domain subjected to a strong external heat source, Boundary Elements Techniques, Tsinghua University Press, Springer, 2002, 377-382.

[6] Jasiński M., Pownuk A., Modelling of heat transfer in biological tissue by interval FEM, Computer Assisted Mechanics and Engineering Sciences 2000, 7(4), 551-558.

[7] Jasiński M., Investigation of tissue thermal damage process with application of direct sensitivity method, Mol. Cell. Biomech. 2013, 10(3), 183-199.

[8] Majchrzak E., Dziatkiewicz G., Paruch M., The modelling of heating a tissue subjected to external electromagnetic field, Acta Bioeng. Biomech. 2008, 10(2), 29-37.

[9] Jasiński M., Modelling of 1D bioheat transfer with perfusion coefficient dependent on tissue necrosis, Scientific Research of the Institute of Mathematics and Computer Science 2009, 7, 57-62.

[10] Majchrzak E., Application of different variants of the BEM in numerical modeling of bioheat transfer processes, Mol. Cell. Biomech. 2013, 10(3), 201-232.

[11] Jasiński M., Numerical modelling of tissue coagulation during laser irradiation controlled by surface temperature, Scientific Research of the Institute of Mathematics and Computer Science 2010, 9, 29-36.

[12] Majchrzak E., Kałuża G., Poteralska J., Application of the DRBEM for numerical solution of Cattaneo-Vernotte bioheat transfer equation, Scientific Research of the Institute of Mathematics and Computer Science 2008, 7, 11-120.

[13] Xu F., Seffen K.A., Lu T.J., Non-Fourier analysis of skin biothermomechanics, Int. J. Heat Mass Transfer 2008, 51(9-10), 2237-2259.

[14] Majchrzak E., Turchan L., The general boundary element method for 3D dual-phase lag model of bioheat transfer, Eng. Anal. Bound. Elem. 2015, 50, 76-82. 